\title{
Analysis of multiple cytokines in aqueous humor of patients with idiopathic macular hole
}

\author{
Heping Wang ${ }^{1}$, Yuqi $\mathrm{Li}^{2,3}$, Song Han ${ }^{1}$ and Tongtong Niu${ }^{1 *}$
}

\begin{abstract}
Background: Idiopathic macular holes are common ophthalmic manifestations with unknown pathogenesis. Thus far, there has been minimal research regarding the causes of idiopathic macular holes, especially with respect to the underlying immune mechanism. To provide clarity regarding the treatment and prognosis of idiopathic macular holes, specifically regarding the levels of cytokines in affected patients, this study examined and analyzed multiple cytokine levels in aqueous humor from patients with idiopathic macular holes.
\end{abstract}

Methods: This comparative cross-sectional study included 38 patients in two groups: a cataract control group $(n=$ 17) and an idiopathic macular hole group $(n=21)$. The levels of 48 cytokines in aqueous humor were detected by multiplex analysis with antibody-coupled magnetic beads. The Kolmogorov-Smirnov test was used to check whether the data were normally distributed; Student's t-test and the Mann-Whitney $U$ test were used to assess differences in cytokine levels between the two groups. Spearman correlation analysis was used to assess relationships among cytokine levels in the experimental group. Signaling pathways containing cytokines with significantly different expression in the experimental group were identified.

Results: There were significant differences in aqueous humor cytokine levels between patients with idiopathic macular holes and patients in the cataract control group. Notably, hepatocyte growth factor $(p=0.0001)$, GM-CSF $(p=0.0111)$, and IFN- $\gamma(p=0.0120)$ were significantly upregulated in the experimental group, while TNF- $a(p=$ 0.0032), GRO-a $(p<0.0001)$, and MIF ( $p<0.0001)$ were significantly downregulated in the experimental group. Furthermore, the GM-CSF level showed significant positive correlations with levels of IL-1 $(r=0.67904, p<0.001)$, IL$4(r=0.76017, p<0.001)$, and IFN- $\gamma(r=0.59922, p=0.004097)$ in the experimental group. Moreover, the levels of nerve growth factor and hepatocyte growth factor showed a significant positive correlation $(r=0.64951, p=$ 0.001441 ) in the experimental group.

Conclusions: Patients with idiopathic macular holes showed significant variation in aqueous humor immune response after the onset of hole formation, including the recruitment of immune cells and regulation of cytokine expression. Our findings also suggest that it is not appropriate to use patients with macular holes as the control group in studies of aqueous humor cytokine levels in ophthalmic diseases.

Keywords: Macular hole, Cytokines, Data analysis

\footnotetext{
* Correspondence: tongtongniu968@hotmail.com

'Department of Ophthalmology, The Fourth Hospital of Shenyang, 110016

Shenyang, Liaoning, People's Republic of China

Full list of author information is available at the end of the article
}

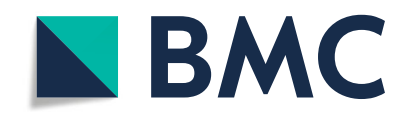

(c) The Author(s). 2021 Open Access This article is licensed under a Creative Commons Attribution 4.0 International License, which permits use, sharing, adaptation, distribution and reproduction in any medium or format, as long as you give appropriate credit to the original author(s) and the source, provide a link to the Creative Commons licence, and indicate if changes were made. The images or other third party material in this article are included in the article's Creative Commons licence, unless indicated otherwise in a credit line to the material. If material is not included in the article's Creative Commons licence and your intended use is not permitted by statutory regulation or exceeds the permitted use, you will need to obtain permission directly from the copyright holder. To view a copy of this licence, visit http://creativecommons.org/licenses/by/4.0/. The Creative Commons Public Domain Dedication waiver (http://creativecommons.org/publicdomain/zero/1.0/) applies to the data made available in this article, unless otherwise stated in a credit line to the data. 


\section{Background}

Macular holes constitute tissue defects that extend from the retinal inner membrane to the photoreceptor layer of the macula, seriously impairing central vision in affected patients. Kuhnt first reported non-traumatic macular holes in 1900 [1]. Since then, various causes of macular holes have been recognized [2]. The overall prevalence of macular holes is $0.33 \%$; affected patients most commonly exhibit agnogenic idiopathic macular holes (approximately 83\% of patients with macular holes). Moreover, macular holes most frequently occur in healthy women aged $>50$ years (mean age, 65 years; female: male ratio of $2: 1) ; 6-28 \%$ of affected patients have bilateral macular holes [3].

Idiopathic macular holes (IMHs) have no obvious systemic or ocular local causes, and do not involve disease in the fundus itself. Current research suggests that IMHs are mainly associated with age-related degenerative changes in the vitreoretinal interface. In recent years, IMHs have become increasingly important in clinical practice because of the large number of older people in many countries, and the morbidity of this condition has gradually increased [4].

The pathogenesis of IMHs has been unclear. In 1995, Gass proposed a theory of posterior vitreous tangential traction [5]. Based on this theory, vitrectomy combined with internal limiting membrane stripping became a common surgical procedure for the treatment of IMHs, although this theory was unable to explain the molecular mechanism underlying IMH formation. Investigation of this mechanism is important for preventing the occurrence of IMHs [6].

In prior studies involving histological analysis of the internal limiting membrane in patients with IMHs, components of Müller cells, glial cells, and fibroblasts were identified [7, 8]. The present analysis of cytokines in aqueous humor was performed to investigate whether intraocular cell proliferation is mediated by inflammatory and immune cells, as well as cytokines secreted by these cells, during the occurrence, development, and repair of IMHs.

\section{Methods}

\section{Participants and ethics approval}

The experimental group in this study included 21 eyes of 21 patients ( 1 man and 20 women) with stage $\geq 3$ IMHs from April 2018 to April 2019. The control group comprised 17 eyes of 17 non-diabetic patients who underwent cataract surgery at the same time, including 12 men and 5 women. The inclusion criteria in both groups were no other retinal or optic neuropathy, except diffuse retinopathy. Exclusion criteria included (1) any other eye disease (e.g., glaucoma or uveitis), (2) history of ocular surgery, and (3) history of ocular inflammation.
The baseline characteristics of patients with IMHs $(n=$ $21)$ and controls $(n=17)$ are shown in Table 1. Participants were considered to have hypertension if their blood pressure was above $140 / 90 \mathrm{mmHg}$ or they were taking any antihypertensive medications. Participants were considered to have hypercholesterolemia if their total fasting plasma cholesterol level was $>200 \mathrm{mg} / \mathrm{dl}$. Participants were considered to have hypertriglyceridemia if their fasting plasma triglycerides level was > $200 \mathrm{mg} / \mathrm{dl}$. Participants were considered to be nonsmokers (no smoking for at least 1 year) or current smokers. The study was approved by the Ethics Committee of the Fourth People's Hospital of Shenyang, People's Republic of China, and followed the tenets of the Declaration of Helsinki. All patients provided written informed consent prior to participation in the study.

\section{Diagnosis and surgery}

This study was performed using a comparative crosssectional method at the Fourth People's Hospital of Shenyang (People's Republic of China). None of the patients in the IMH group had cataracts; none of the patients in the cataract group had IMHs. All patients in the IMH group underwent complete physical and ophthalmologic examination for clinical diagnosis of IMHs, including assessments of visual acuity and relative afferent pupillary defect, as well as multifocal electroretinography, optical coherence tomography, fundus examination, and fluorescent fundus angiography. During cataract surgery, limbal puncture was performed with a sterile tuberculin syringe. An undiluted aqueous sample $(0.2-0.5 \mathrm{ml})$ was drawn into the syringe, then transferred to a $2-\mathrm{ml}$ centrifuge tube. Immediately after the end of the operation, $0.2-0.5 \mathrm{ml}$ aqueous solution was extracted by anterior chamber puncture. All samples were snapfrozen in liquid nitrogen and stored at $-80{ }^{\circ} \mathrm{C}$ until analysis.

Table 1 Baseline characteristics of patients with IMHs $(n=21)$ and patients with cataracts $(n=17)$

\begin{tabular}{llll}
\hline Characteristics & IMH & Cataract & $P$ value \\
\hline Number & 21 & 17 & - \\
Gender & & & $<0.01^{\mathrm{a}}$ \\
Male (\%) & 1 & 12 & \\
Female (\%) & 20 & 5 & \\
Age (SD) & 66.7 & 69.3 & $0.159^{\mathrm{b}}$ \\
Hypertension (\%) & 76 & 40 & $0.062^{\mathrm{a}}$ \\
Body mass index (SD) & 29.62 & 27.32 & $0.061^{\mathrm{b}}$ \\
Smoking (\%) & 42 & 25 & $0.366^{\mathrm{a}}$ \\
\hline
\end{tabular}

${ }^{\mathrm{a}}$ Pearson $\mathrm{x}^{2}$ test. $^{\mathrm{b}}$ Student's t-test 


\section{Measurement of aqueous cytokine levels}

A Bio-Plex Pro Human Cytokine 48-plex Screening Kit (Bio-Rad, Hercules, CA, USA) was used to determine the levels of 48 human cytokines in the collected aqueous samples (Table 2).

All experimental measurements were performed in accordance with the kit manufacturer's instructions. Briefly, $50 \mu$ of $1 \times$ beads were added to each well and the beads were washed twice with $200 \mu \mathrm{l}$ of wash buffer per wash. Fifty-microliter aliquots of standards, samples, and controls (i.e., negative controls that are provided with the test kit) were added to respective wells. Plates were incubated in a shaker at $850 \mathrm{rpm}$ for $30 \mathrm{~min}$ at room temperature. Each well was then washed three times with $100 \mu$ of wash buffer per wash. Twenty-five microliters of $1 \times$ detection antibody was added to each well and the plates were incubated in a shaker at $850 \mathrm{rpm}$ for $30 \mathrm{~min}$ at room temperature. Each well was then washed three times with $100 \mu \mathrm{l}$ of wash buffer per wash. Fifty microliters of $1 \times$ streptavidin-PE was added to each well and the plates were incubated in a shaker at $850 \mathrm{rpm}$ for $10 \mathrm{~min}$ at room temperature. Each well was then washed three times with $100 \mu$ of wash buffer. Samples were resuspended in $125 \mu$ l of assay buffer and the plates were incubated in a shaker at $850 \mathrm{rpm}$ for $30 \mathrm{~s}$ at room temperature.

The measurements were performed in accordance with the kit manufacturer's instructions and data were acquired using the Bio-Plex TM 200 system, software version 6.0 (Bio-Rad). The standard curve for each cytokine was generated using the reference set of cytokine concentrations provided with the kit; sample concentrations of each cytokine were calculated using the multiparameter standard curve. If a sample concentration was above or below the detection limit, it was considered and outlier and removed from analysis.

\section{Statistical analysis}

Data are shown as mean \pm standard deviation, range, median, and IQR. Statistical analysis was performed using $R$, version 3.6.1 ( $R$ Foundation for Statistical Computing, Vienna, Austria). The Kolmogorov-Smirnov test was used to determine whether the data were normally distributed. The Pearson $\chi^{2}$ test was used for comparisons of qualitative variables. Student's $t$ test and the Mann-Whitney $U$ test were used for comparisons of quantitative variables between two groups. Spearman correlation analysis was used to assess relationships among cytokine levels in the experimental group. $p$-values $<0.05$ were considered statistically significant. Pathway enrichment analysis (http://metascape.org) was used to identify major signaling pathways that contained cytokines with significantly altered expression between groups.

\section{Results \\ Cytokine levels in aqueous humor}

Levels of cytokines in aqueous humor samples were compared between the IMH and control groups (Table 2). Figure 1 shows a visual comparison of all tested cytokines between the two groups. Twenty-seven cytokines with significant differences in levels are shown in Fig. 2a; of these 27, 20 were significantly upregulated and seven were significantly downregulated. Significantly upregulated cytokines included IL-1r $\alpha$ $(p=0.0038)$, IL-4 $(p=0.0024)$, IL-13 $(p=0.0017)$, IL-15 $(p=0.0066), \mathrm{IL}-1 \alpha(p=0.0027), \mathrm{IL}-2 \mathrm{R} \alpha(p=0.0287), \mathrm{IL}-$ $3(p=0.0014), \mathrm{IL}-12 \mathrm{p} 40(p=0.0028), \mathrm{IL}-18(p=0.0003)$, hepatocyte growth factor (HGF) $(p=0.0001)$, GM-CSF $(p=0.0111)$, IFN- $\gamma \quad(p=0.0120)$, MIP-1 $\alpha \quad(p=0.0030)$, PDGF-bb $(p=0.0027)$, LIF $(p=0.0006), \operatorname{M-CSF}(p<$ $0.0001), \beta-N G F(p=0.0004)$, SDF- $1 \alpha(p=0.0130)$, TNF$\beta(p<0.0001)$, and TRAIL $(p=0.0001)$. Significantly downregulated cytokines included IL-5 $(p=0.0208)$, eotaxin $(p<0.0001)$, IP-10 $(p=0.0001)$, TNF- $\alpha \quad(p=$ $0.0032)$, GRO $\alpha(p<0.0001)$, IL-7 $(p=0.0367)$, and MIF $(p<0.0001)$. Cytokines that were significantly upregulated and might be closely related to IMHs are shown in Fig. 2b.

\section{Relationships among cytokine levels}

Table 3 shows the relationships among cytokines that were significantly different between the IMH and control groups. Notably, the level of GM-CSF was significantly positively correlated with the levels of inflammationrelated cytokines including IL-1 $(r=0.67904, p<0.001)$, IL-4 $(r=0.76017, p<0.001)$, and IFN- $\gamma(r=0.59922, p=$ $0.004097)$ both. In addition, the level of neuronal and tissue repair-related nerve cell growth factor (NGF) was significantly positively correlated with the level of HGF $(r=0.64951, p=0.001441)$.

\section{Exploration and analysis of cytokine function}

Figure 3 shows the results of pathway enrichment analysis (http://metascape.org) involving cytokines that were significantly different between the IMH and control groups. The greatest proportion of cytokines was involved in the cytokine-cytokine receptor pathway, which is implicated in inflammatory responses. This includes both members of pro-inflammatory signaling pathways (e.g., IL-17, IL-14, and IL-13) and members of anti-inflammatory signaling pathways (e.g., IL-10). Moreover, the cytokine levels in hematopoietic lineage signaling pathways associated with tissue repair exhibited significant differences between groups. 


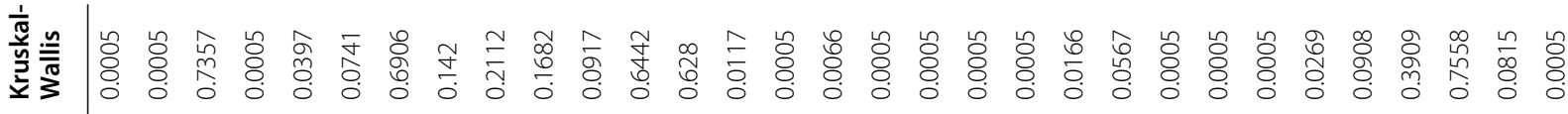

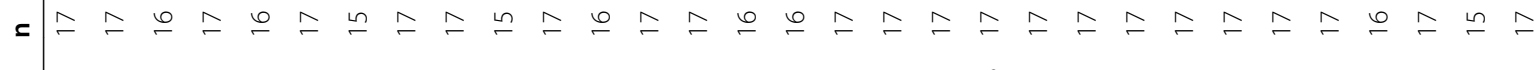

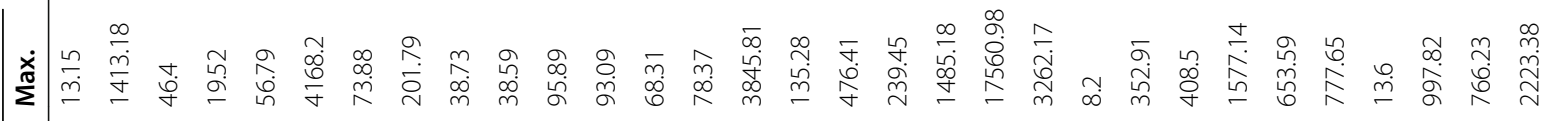

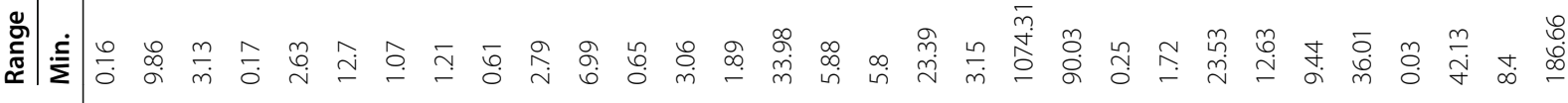

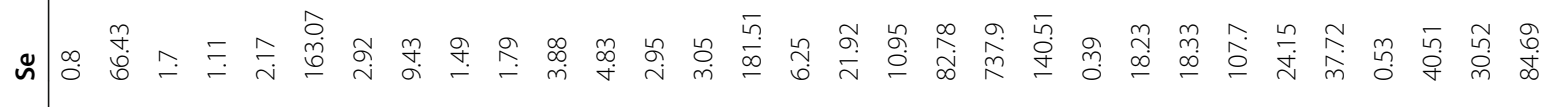

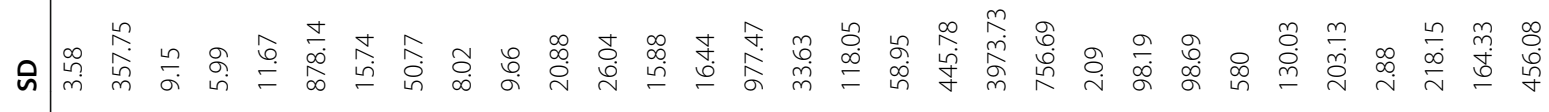

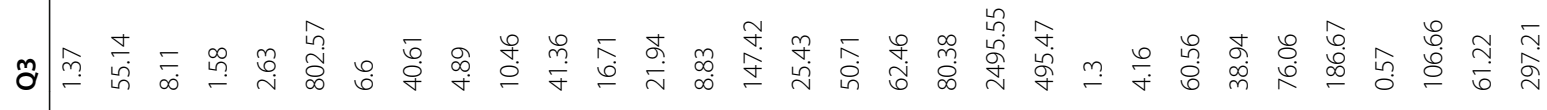

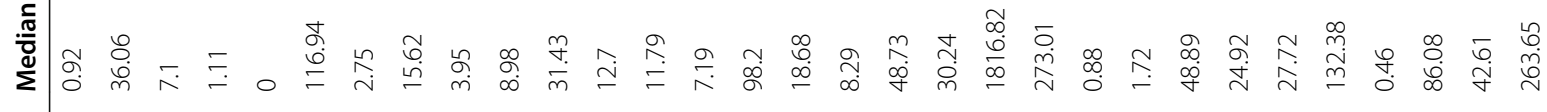

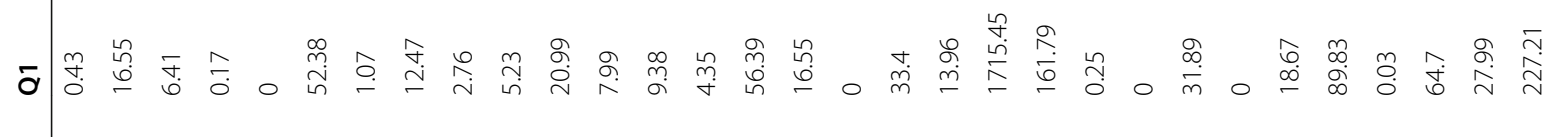

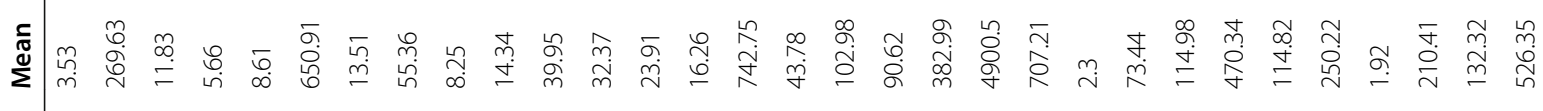

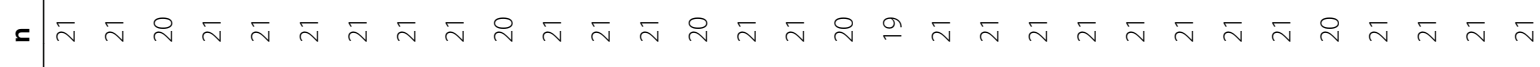

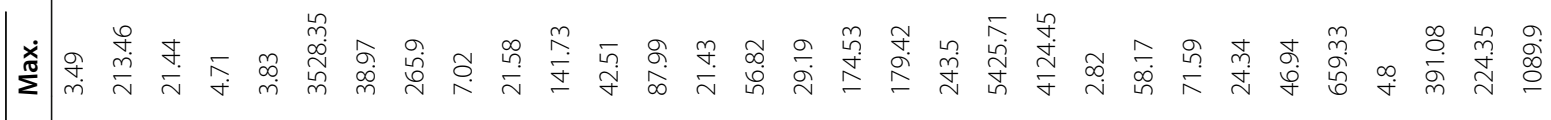

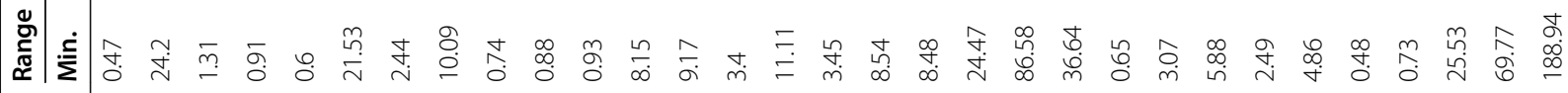
๗

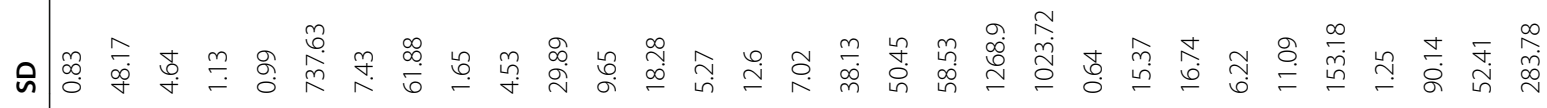

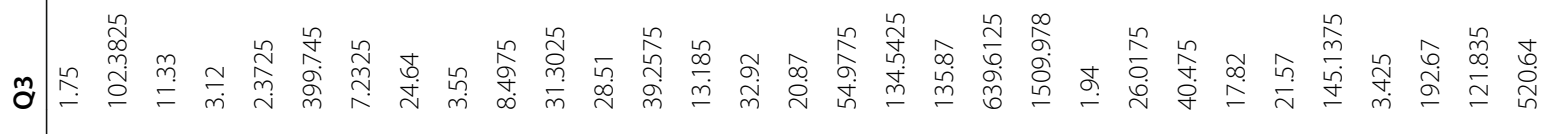

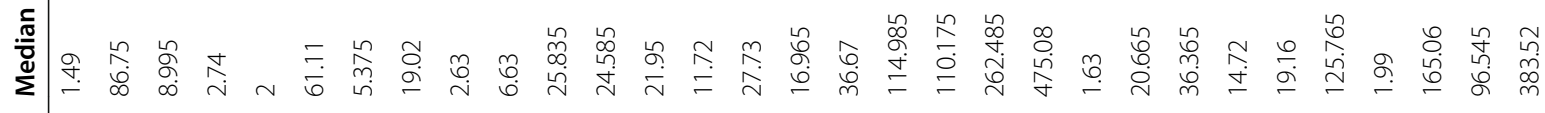

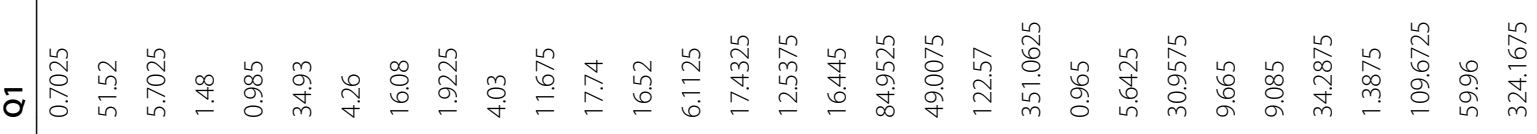

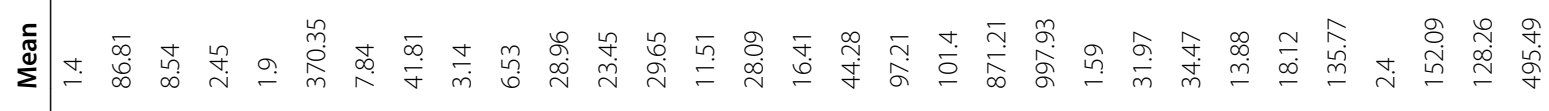

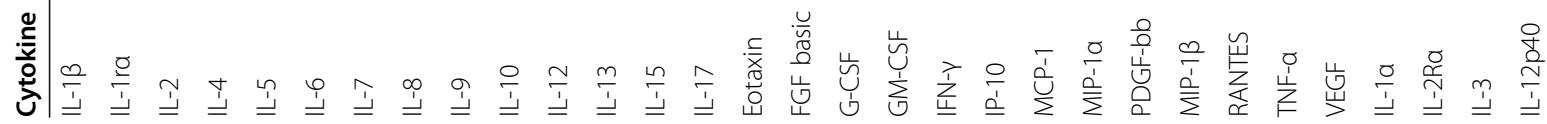




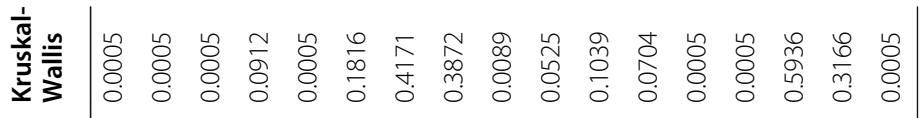

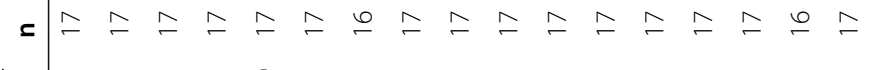

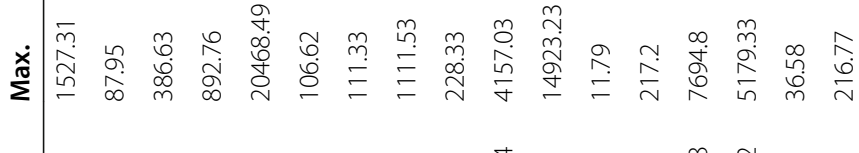

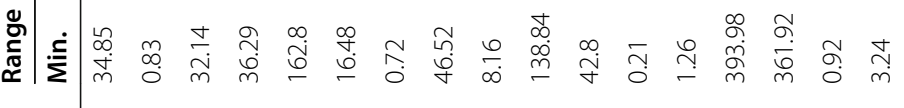

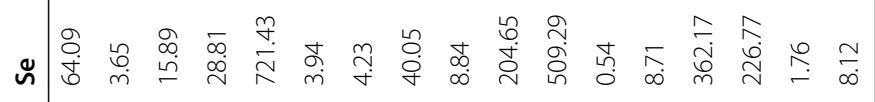

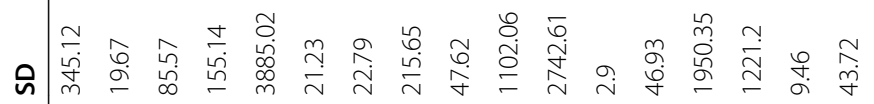

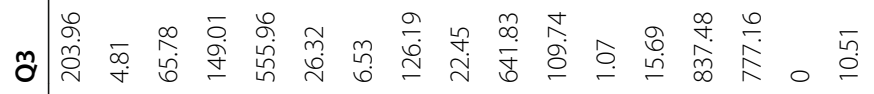

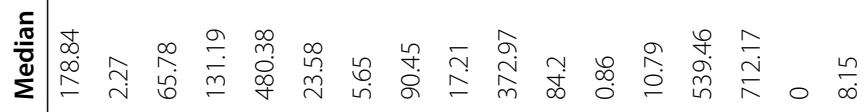

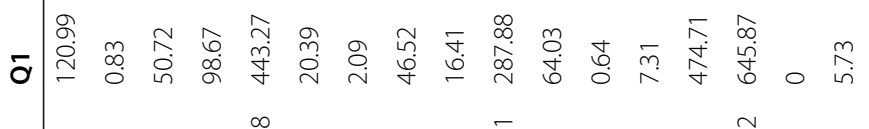

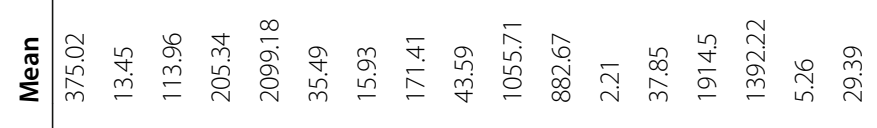

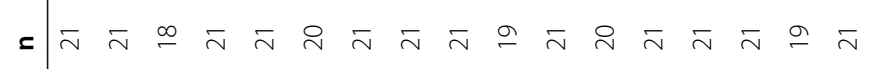

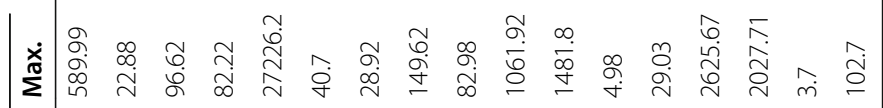

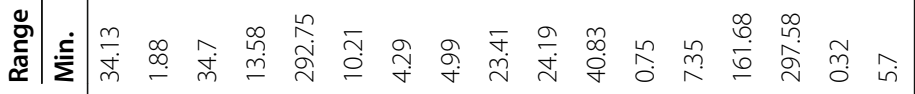

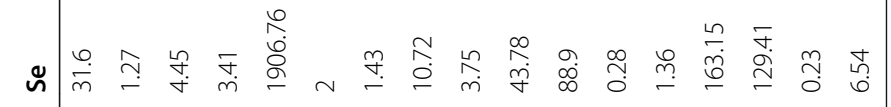

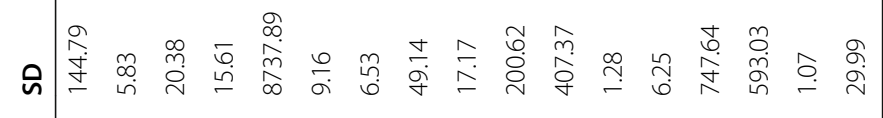

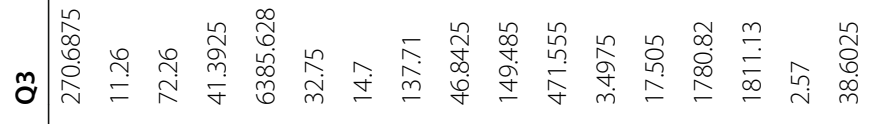

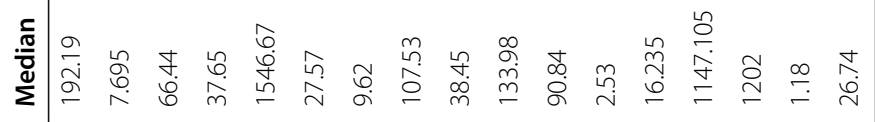

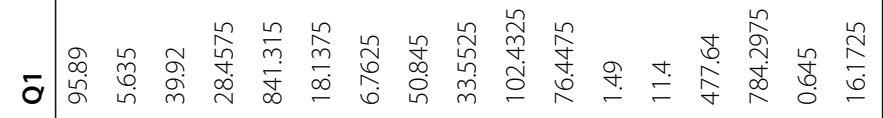

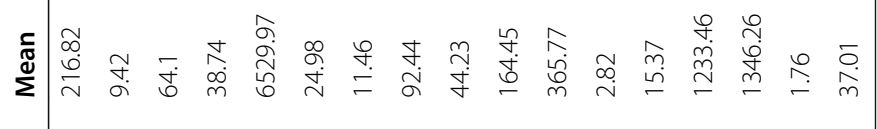

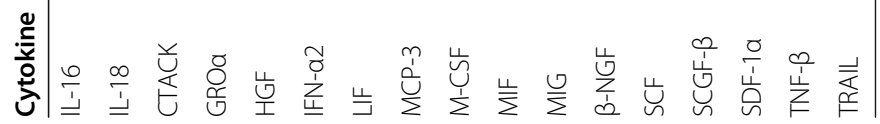



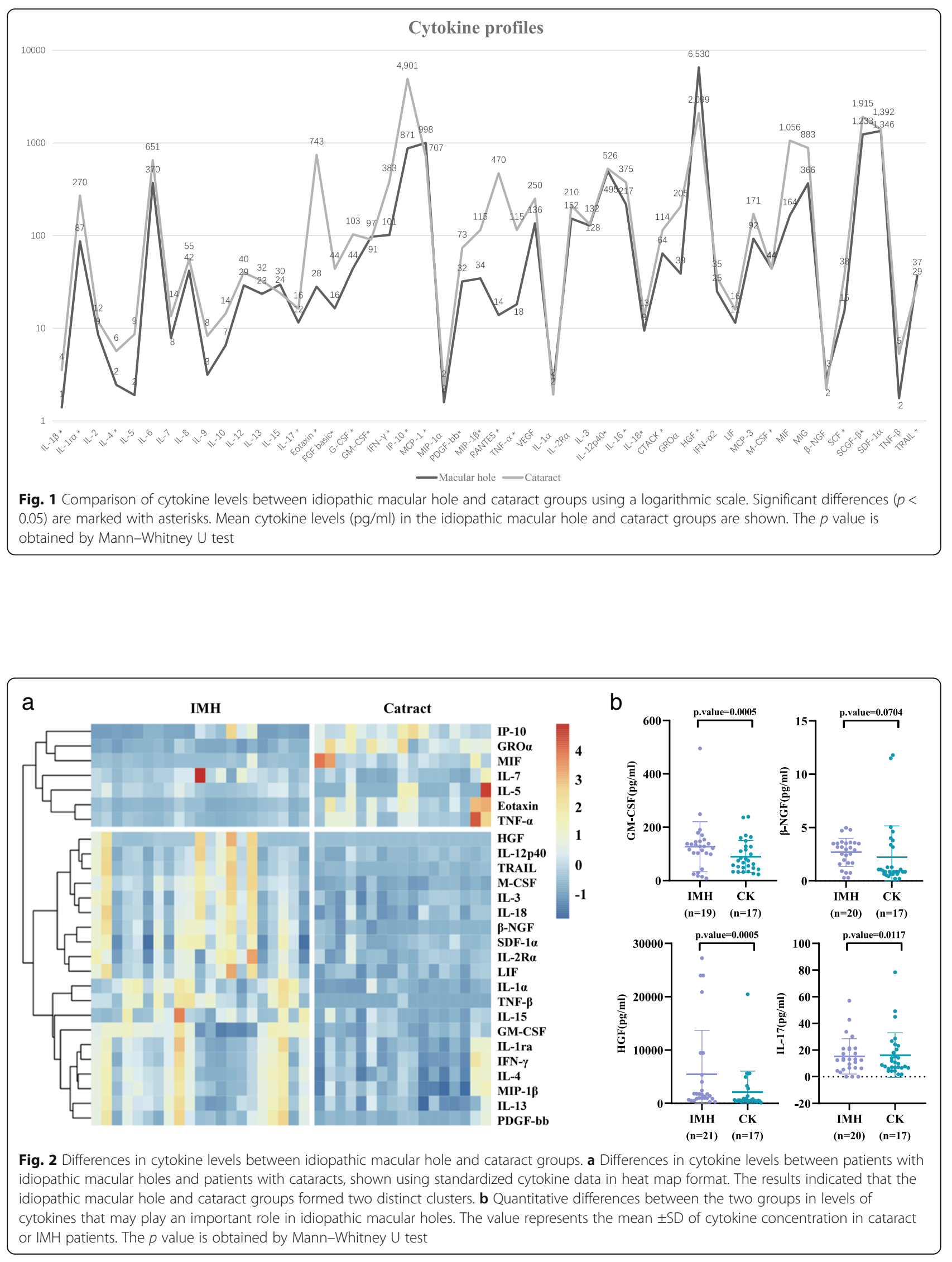


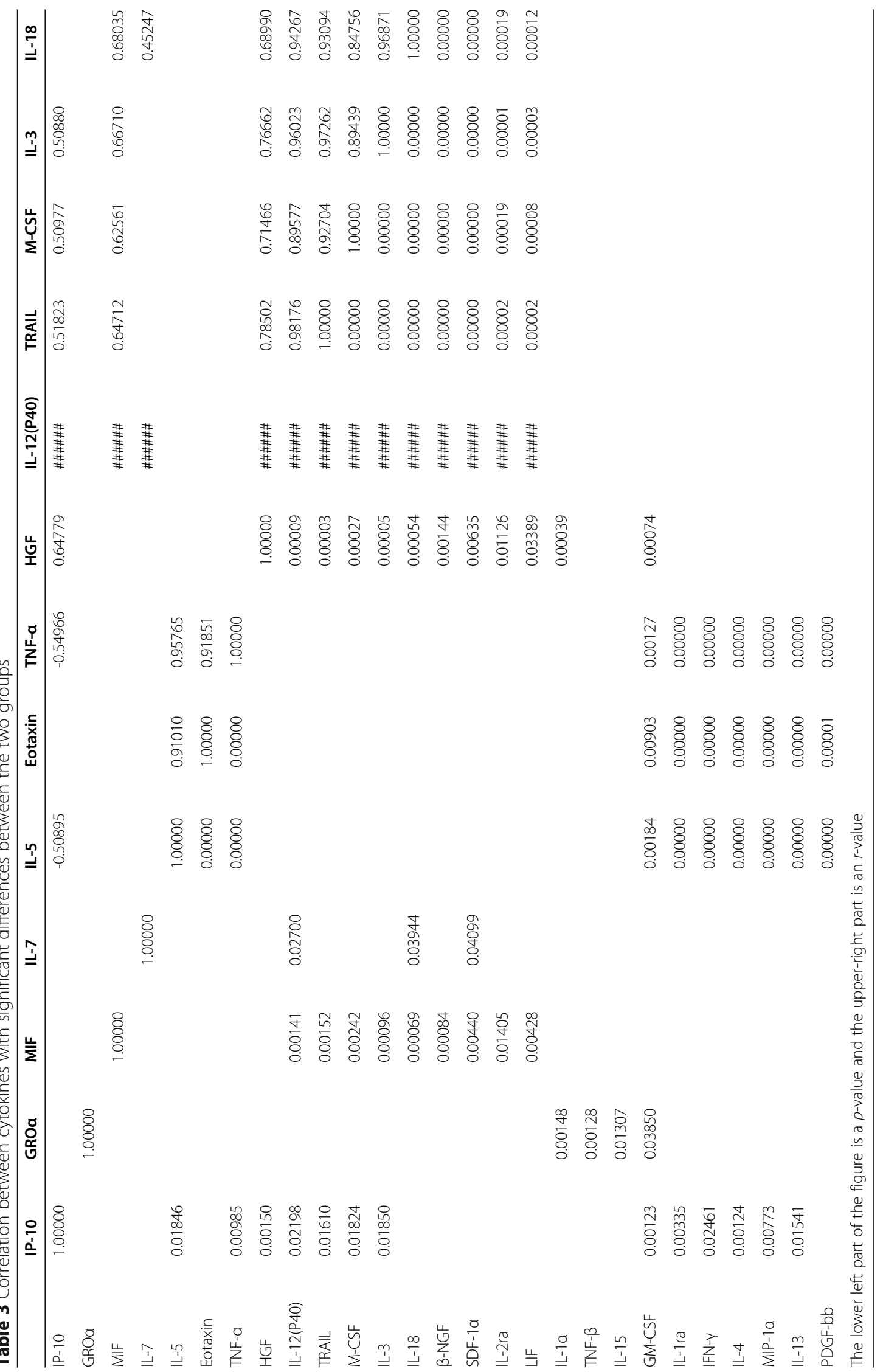




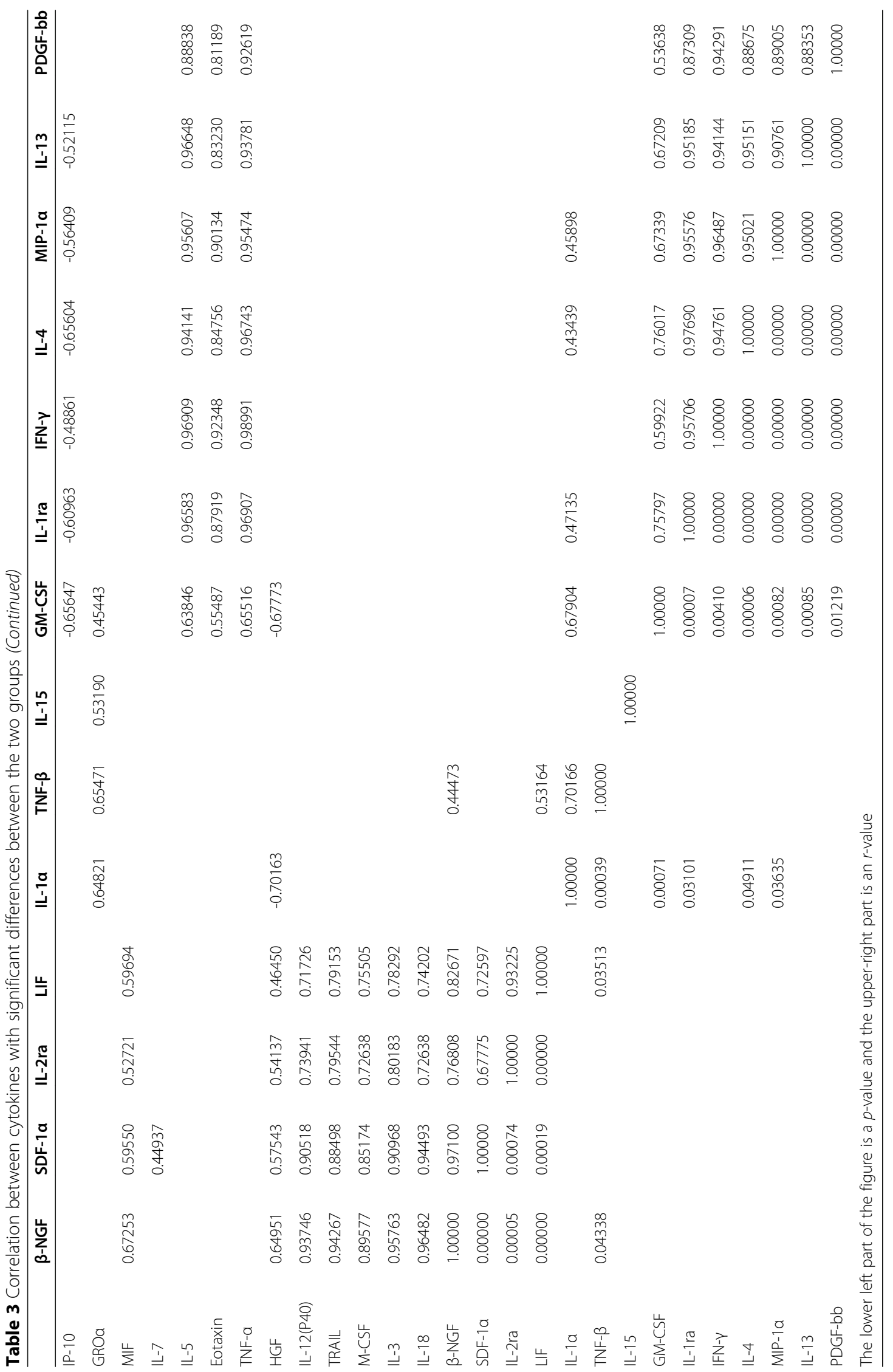




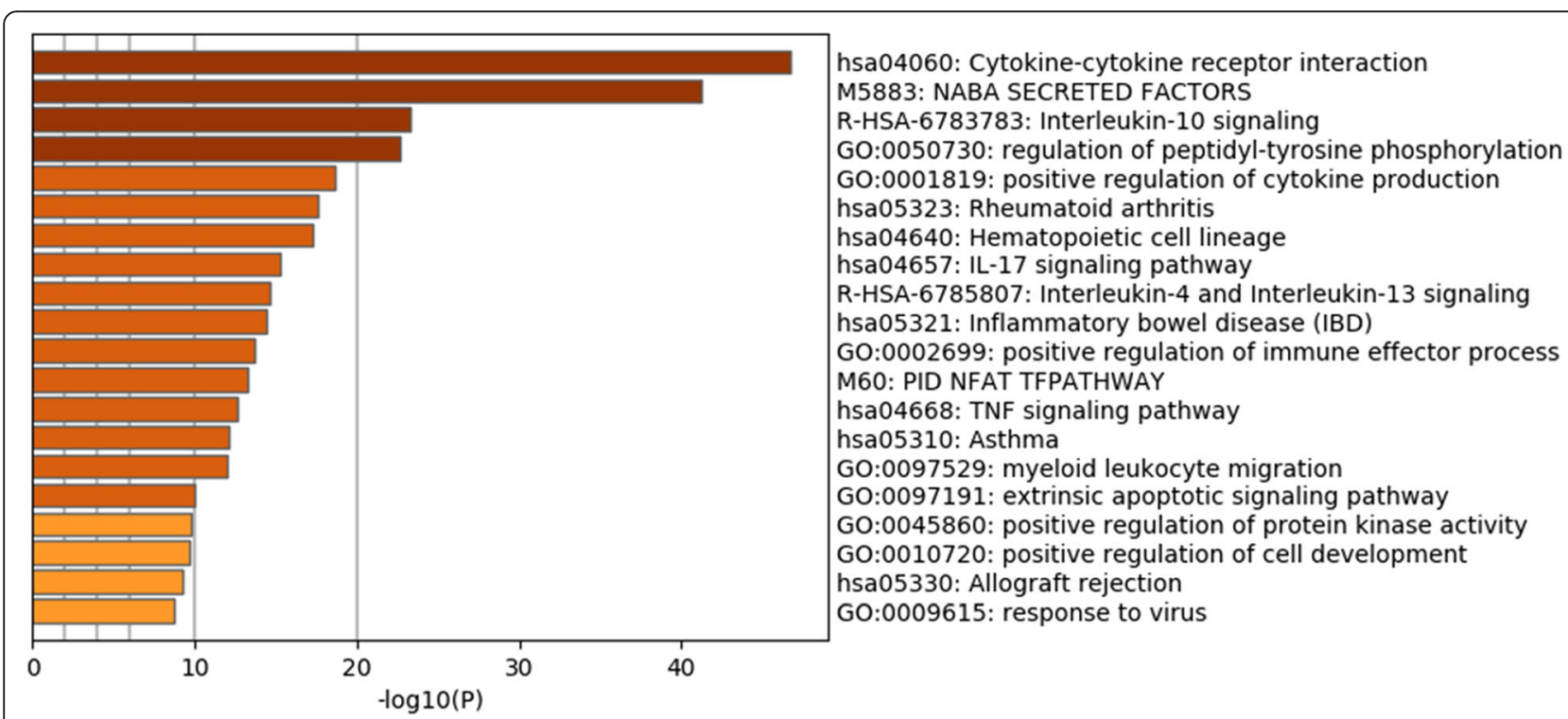

Fig. 3 Signal pathway analysis of cytokines with significant differences in levels between the two groups. A greater value on the horizontal axis indicates greater magnitude of the between-group difference in cytokine levels involved in this pathway

\section{Discussion}

IMH etiology or stage of development is generally considered to be unrelated to ocular inflammation; thus, affected patients are often included as a control group in analyses of inflammation-related ocular diseases [9]. However, after onset of IMHs, tissue damage and reparation often lead to local inflammatory reactions, which affect cytokine levels in the aqueous humor. The degree of ocular inflammatory response and changes in cytokine levels in the aqueous humor provide useful information for the treatment, prognosis, and diagnosis of IMH.

In the normal retina, Müller cells penetrate the neurosensory layer; the fibers of these cells extend from the outer membrane to the inner limiting membrane, thereby providing retinal support and nutrient metabolism. After IMH formation, the retina undergoes a process called reactive gliosis [10]. This event is triggered by penetration damage involving activated astrocytes. The activation of intermediate filament proteins (i.e., glial fibrillary acidic protein and vimentin) is critical for the formation of glial scars $[11,12]$.

In the retina, Müller cells are the major glial cell type involved in reactive scar formation following diseaseinduced retinal degeneration [13]. To restore nutrients, Müller cells and other glial cells in the retina may migrate to the outer surface of the inner limiting membrane in response to various inflammatory factors. Some researchers have proposed that proliferation and contraction of these cells in the inner limiting membrane results in IMH progression. After IMH formation, lymphocytes can destroy the blood-retina barrier and migrate into the vitreous cavity; here, lymphocytes make contact with transitional pigment cells and glial cells, secrete cytokines, promote an inflammatory response, and enhance macrophage-mediated phagocytosis [14]. In addition, lymphocytes in the vitreous cavity can promote the transformation of glial cells into fibroblasts and accelerate the repair of tissue hyperplasia [15].

Immune system-mediated inflammatory responses are known to play important roles in injury-induced neurological damage and tissue repair. The inflammatory response during trauma is mainly mediated by innate immune cells including microglia and astrocytes, which play important roles. However, the recruitment of these innate immune cells leads to some recruitment of $\mathrm{T}$ cells and other immune cells and subsequently induces apoptosis and damage repair [16].

The results of this study showed significant upregulation of GM-CSF, which is an important component of microglial products. Microglia represent a type of macrophage; thus, they can phagocytose damaged cell debris and release various cytokines. In IMHs, the microglia in the damaged macular area are rapidly activated, thereby causing microglia morphology to transform into an "ameboid-like" state and migrate to the site of injury. This is followed by the release of various cytokines including NGF, IL-4 (a protective cytokine), IL-10 (an immunosuppressive cytokine), as well as NO and ROS; pro-inflammatory cytokines are also produced (e.g., IL-1, IL-6, and IFN- $\gamma$ ) [17]. These cytokines might accumulate in the aqueous humor, as shown in our study. The upregulation of GM-CSF, IL-1, IL-4, and 
IFN- $\gamma$ indicates that microglia play an important role in the immune response during IMH formation and progression.

Previous studies of cytokine levels in the vitreous of patients with ERM and patients with IMHs revealed no significant difference in GM-CSF levels between the two groups. Conversely, we found that the GM-CSF level was significantly upregulated in patients with IMHs, which implies that the use of patients with IMHs as the control group may lead to inaccurate results [18].

The significant downregulation of MIF level in the aqueous extract of patients with IMHs suggests that monocytes play important roles in the activation and development of Müller cells after the onset of IMH formation. The significant upregulation of IL-3, M-CSF, GM-CSF, and IFN- $\gamma$ levels supported the notion that monocytes play important roles in IMHs. Notably, the MIF level was negatively correlated with the LIF level; LIF is an important neurogenic factor that could promote the differentiation of glial cells. LIF is mainly secreted by macrophages, which implies that macrophages play an important role in the body's self-repair process after IMH onset.

Microglia activation and production of IL-1 cause activation of astrocytes and Müller cells. During the adaptive nerve injury in patients with IMHs, Müller cells can produce cytokines (e.g., NGF and HGF) to promote neuron survival. In this study, both NGF and HGF levels were significantly upregulated in comparison with the control group, and the NGF level was associated with the HGF level. Because HGF and NGF both play important roles in the treatment of ocular nerve injury diseases (e.g., corneal damage and glaucoma), we hypothesized that HGF and NGF also play important roles in tissue repair during the prophase and postoperative recovery phase of IMHs [19].

In this study, a multiplex analysis with antibodycoupled magnetic beads was used to detect changes in cytokine levels in aqueous humor samples from patients with IMHs. Compared with the traditional ELISA method, this method can detect a wider variety of cytokines with lower sample volume. Moreover, the method can simultaneously detect 48 cytokines in a single experiment, thus avoiding instability related to batch effects [20].

\section{Conclusions}

This study demonstrated significant changes in the levels of various cytokines in the aqueous humor between patients with IMHs and patients with cataracts, presumably in relation to the roles of microglia and astrocytes in the pathogenesis and repair of IMHs. In addition, HGF and NGF were significantly upregulated, as were other cytokines that promote nerve growth and survival; these findings can aid in the treatment and prognosis of IMHs. Notably, patients with IMH are commonly used as the control group in research regarding aqueous humor cytokine levels in ophthalmic diseases. The results of this study suggest that the levels of multiple cytokines in the aqueous humor change considerably due to the inflammatory response in patients with IMHs. Therefore, it may not be appropriate to use patients with IMHs as a control group in studies of aqueous humor cytokine levels.

\section{Abbreviation \\ $\mathrm{IMH}$ : Idiopathic macular hole}

\section{Acknowledgements}

We thank Ryan Chastain-Gross, Ph.D., from Liwen Bianji, Edanz Group China (www.liwenbianji.cn/ac), for editing the English text of a draft of this manuscript.

\section{Authors' contributions}

Examined the aqueous humor data: SH, TTN. Analyzed the data, and was a major contributor in writing the manuscript: HPW, YQL. All authors read and approved the final manuscript.

\section{Funding}

This research was supported by Science and Technology Innovation Projects of Shenyang City (18-014-4-63). Corresponding funds were used to purchase experimental materials, use instruments, and seek technical supports.

\section{Availability of data and materials}

The datasets generated and analyzed during the current study are not publicly available due Follow-up research needs but are available from the corresponding author on reasonable request.

\section{Ethics approval and consent to participate}

Approval for the collection and research of human aqueous humor was permitted by the Ethics Committee of the Fourth People's Hospital of Shenyang, People's Republic of China (ID 18-014-4-63). All patients were treated in accordance with the Declaration of Helsinki, and written informed consents were obtained from all participants prior to their participation in the study.

Consent for publication

Not applicable.

\section{Competing interests}

The authors declare that they have no competing interests.

\section{Author details}

'Department of Ophthalmology, The Fourth Hospital of Shenyang, 110016 Shenyang, Liaoning, People's Republic of China. ${ }^{2}$ University of Chinese Academy of Sciences, Beijing, People's Republic of China. ${ }^{3}$ Institute of Applied Ecology, Chinese Academy of Sciences, Shenyang, Liaoning, People's Republic of China.

Received: 30 April 2020 Accepted: 21 December 2020 Published online: 11 January 2021

\section{References}

1. Aaberg TM, Blair CJ, Gass JD. Macular holes. Am J Ophthalmol. 1970;69(4): 555-62.

2. Kang SW, Ahn K, Ham DI. Types of macular hole closure and their clinical implications. Br J Ophthalmol. 2003;87(8):1015-9.

3. Ho AC, Guyer DR, Fine SL. Macular hole. Survey of ophthalmology. 1998; 42(5):393-416

4. Smiddy WE, Flynn HW Jr. Pathogenesis of macular holes and therapeutic implications. Am J Ophthalmol. 2004;137(3):525-37. 
5. Gass JD. Reappraisal of biomicroscopic classification of stages of development of a macular hole. Am J Ophthalmol. 1995;119(6):752-9.

6. Gass JD. Idiopathic senile macular hole. Its early stages and pathogenesis. Archives of ophthalmology (Chicago III: 1960). 1988;106(5):629-39.

7. Brooks HL Jr. Macular hole surgery with and without internal limiting membrane peeling. Ophthalmology. 2000;107(10):1939-48.

8. Terasaki H, Miyake Y, Nomura R, Piao CH, Hori K, Niwa T, Kondo M. Focal macular ERGs in eyes after removal of macular ILM during macular hole surgery. Investig Ophthalmol Vis Sci. 2001;42(1):229-34.

9. Loukovaara S, Sandholm J, Aalto K, Liukkonen J, Jalkanen S, Yegutkin GG Deregulation of ocular nucleotide homeostasis in patients with diabetic retinopathy. J Mol Med. 2017;95(2):193-204.

10. Uemoto R, Yamamoto S, Takeuchi S. Epimacular proliferative response following internal limiting membrane peeling for idiopathic macular holes. Graefe's archive for clinical experimental ophthalmology = Albrecht von Graefes Archiv fur klinische experimentelle Ophthalmologie. 2004;242(2): 177-80.

11. Vogt D, Haritoglou C, Mautone L, Hagenau F, Guenther SR, Wolf A, Priglinger SG, Schumann RG: Premacular Cells as Source of Neurotrophic Factors in Idiopathic Macular Holes. Curr Eye Res. 2020;45(11):1395-402.

12. Lesnik Oberstein SY, Lewis GP, Dutra T, Fisher SK. Evidence that neurites in human epiretinal membranes express melanopsin, calretinin, rod opsin and neurofilament protein. Br J Ophthalmol. 2011;95(2):266-72.

13. Graca AB, Hippert C, Pearson RA. Muller Glia Reactivity and Development of Gliosis in Response to Pathological Conditions. Adv Exp Med Biol. 2018; 1074:303-8.

14. Musada GR, Dvoriantchikova G, Myer C, Ivanov D, Bhattacharya SK, Hackam AS: The effect of extrinsic Wnt/ $\beta$-catenin signaling in Muller glia on retinal ganglion cell neurite growth. Dev Neurobiol. 2020;80(3-4):98-110.

15. Luo W, Hu L, Li W, Xu G, Xu L, Zhang C, Wang F. Epo inhibits the fibrosis and migration of Müller glial cells induced by TGF- $\beta$ and high glucose. Graefe's archive for clinical experimental ophthalmology = Albrecht von Graefes Archiv fur klinische experimentelle Ophthalmologie. 2016;254(5): $881-90$

16. Zuchero JB, Barres BA. Glia in mammalian development and disease. Development. 2015;142(22):3805-9.

17. Li L, Eter N, Heiduschka P. The microglia in healthy and diseased retina. Exp Eye Res. 2015;136:116-30.

18. Zandi S, Tappeiner C, Pfister IB, Despont A, Rieben R, Garweg JG. Vitreal Cytokine Profile Differences Between Eyes With Epiretinal Membranes or Macular Holes. Investig Ophthalmol Vis Sci. 2016;57(14):6320-6.

19. Miyagi $H$, Thomasy SM, Russell P, Murphy CJ. The role of hepatocyte growth factor in corneal wound healing. Exp Eye Res. 2018;166:49-55.

20. Houser B. Bio-Rad's Bio-Plex(R) suspension array system, xMAP technology overview. Arch Physiol Biochem. 2012;118(4):192-6.

\section{Publisher's Note}

Springer Nature remains neutral with regard to jurisdictional claims in published maps and institutional affiliations.

Ready to submit your research? Choose BMC and benefit from:

- fast, convenient online submission

- thorough peer review by experienced researchers in your field

- rapid publication on acceptance

- support for research data, including large and complex data types

- gold Open Access which fosters wider collaboration and increased citations

- maximum visibility for your research: over $100 \mathrm{M}$ website views per year

At $\mathrm{BMC}$, research is always in progress.

Learn more biomedcentral.com/submissions 